\title{
Tests for Rank Correlation Coefficients. I
}

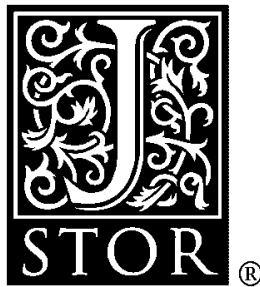

\author{
E. C. Fieller; H. O. Hartley; E. S. Pearson
}

Biometrika, Vol. 44, No. 3/4. (Dec., 1957), pp. 470-481.

Stable URL:

http://links.jstor.org/sici?sici=0006-3444\%28195712\%2944\%3A3\%2F4\%3C470\%3ATFRCCI\%3E2.0.CO\%3B2-6

Biometrika is currently published by Biometrika Trust.

Your use of the JSTOR archive indicates your acceptance of JSTOR's Terms and Conditions of Use, available at

http://www.jstor.org/about/terms.html. JSTOR's Terms and Conditions of Use provides, in part, that unless you have obtained prior permission, you may not download an entire issue of a journal or multiple copies of articles, and you may use content in the JSTOR archive only for your personal, non-commercial use.

Please contact the publisher regarding any further use of this work. Publisher contact information may be obtained at http://www.jstor.org/journals/bio.html.

Each copy of any part of a JSTOR transmission must contain the same copyright notice that appears on the screen or printed page of such transmission.

The JSTOR Archive is a trusted digital repository providing for long-term preservation and access to leading academic journals and scholarly literature from around the world. The Archive is supported by libraries, scholarly societies, publishers, and foundations. It is an initiative of JSTOR, a not-for-profit organization with a mission to help the scholarly community take advantage of advances in technology. For more information regarding JSTOR, please contact support@ jstor.org. 


\title{
TESTS FOR RANK CORRELATION COEFFICIENTS. I
}

\author{
By E. C. FIELLER, H. O. HARTLEY ANd E. S. PEARSON \\ Statistical Advisory Unit, Ministry of Supply, London; \\ Iowa State College, Ames; University College, London
}

\section{Purpose of the Study}

\section{$(1 \cdot 1)$ The measures considered}

The following is a first report on an investigation which became possible with the availability of the 25,000 sets of correlated random normal deviates, 3000 of which were published in Fieller, Lewis \& Pearson's (1955) Tracts for Computers, no. XXVI. The object which we set ourselves was to study with the aid of these data the sampling distributions of, and relationships between, three measures of rank correlation, in the case where the basic variables which have been ranked follow bivariate normal distributions.

We shall use the following notation. Suppose that there are $n$ pairs of associated rankings

$$
u_{1}, u_{2}, \ldots, u_{n} \text { and } v_{1}, v_{2}, \ldots, v_{n},
$$

where the integers $u_{i}(i=1,2, \ldots, n)$ may be taken in ascending order $1,2, \ldots, n$ and the $v_{i}$ are a permutation of these integers. We shall consider in the present paper the two following measures of correlation between these rankings:

(a) Spearman's coefficient which we denote by $r_{S}$. This is simply the product moment correlation coefficient of $u_{i}, v_{i}$ and may be computed from the sum of squared differences

where

$$
S_{S}=\sum_{i=1}^{n}\left(u_{i}-v_{i}\right)^{2}
$$

(b) Kendall's coefficient, $\tau$, which we denote by $r_{K}$. This may be computed as follows. For every integer $u_{i}$ count the number of $v_{j}$ with $v_{j}>u_{i}$ and $j>i$; then add these counts to obtain the positive score $P_{K}$. Then

$$
r_{K}=4 P_{K} /\left(n^{2}-n\right)-1 \text {. }
$$

Both $r_{S}$ and $r_{K}$ lie between +1 and -1 . We shall not be concerned here with ties among the $u$ 's or $v$ 's.

The following is a third coefficient which has been computed for all the sampling data and which we hope to consider later:

(c) The Fisher-Yates coefficient. Let $\xi(i \mid n)$ be a so-called normal order statistic, i.e. the expected value of the $i$ th largest standardized deviate in a sample of $n$ observations from a normal population. Then we may attach these score values to both the $u$ rankings and the $v$ rankings. Fisher \& Yates $(1938$, p. 50) have suggested that a measure of rank correlation might be obtained from the product moment correlation coefficient of these scores, namely

$$
r_{F}=\sum_{i=1}^{n} \xi(i \mid n) \xi\left(v_{i} \mid n\right) / \sum_{i=1}^{n} \xi^{2}(i \mid n) .
$$


Convenient tables of the individual $\xi(i \mid n)$ as well as of $\sum_{i} \xi^{2}(i \mid n)$ are given, for example, in Fisher \& Yates (1938, Tables XX and XXI). As an approximation to the actual productmoment correlation coefficient, $r_{x y}$, in a normal sample $r_{F}$ clearly has much to recommend it; but the only discussions of this coefficient of which we are aware are those by Jeffreys (1948, pp. 209-10) and Hoeffding (1951, pp. 86-9).

\section{$(1 \cdot 2)$ Some known results on the distribution theory of $r_{S}$ and $r_{K}$}

For a comprehensive summary of the older results, the reader may consult Kendall (1948) and Moran (1950). Briefly these are as follows:

For independent random rankings (i.e. for random permutations of the $v_{i}$ ) the complete distributions of $r_{S}$ and $r_{K}$ have been obtained for small $n$ by combinatorial enumeration. Adequate approximations have been evolved for larger $n$.

In the case of correlated rankings it is first necessary to specify the nature of the dependence. A discussion of this problem of appropriate population models was given by Daniels (1950), and very recently Mallows (1957) has developed a new form of approach related to paired-comparison theory. In the present paper we start from the assumption that the $n$ pairs of rankings $u_{i}, v_{i}$ have arisen as the rank numbers in a sample of $n$ pairs of correlated normal variates. Thus, if $x_{i}, y_{i}(i=1,2, \ldots, n)$ denote a random sample of $n$ paired observations from a bivariate normal population having correlation coefficient $\rho$, we suppose that the $x_{i}$ are arranged in order of magnitude and that $v_{i}$ is the rank of $y_{i}$. This model has received considerable attention and a certain number of theoretical results are known. Thus we have

$$
\begin{gathered}
\mathscr{E}\left(r_{S}\right)=\frac{6}{(n+1) \pi}\left\{\sin ^{-1} \rho+(n-2) \sin ^{-1} \frac{1}{2} \rho\right\} \quad(\text { Moran, } 1948), \\
\operatorname{var}\left(r_{S}\right)=1 / n\left\{1-1 \cdot 563465 \rho^{2}+0 \cdot 304743 \rho^{4}+0 \cdot 155286 \rho^{6}+0 \cdot 061552 \rho^{8}+0 \cdot 022099 \rho^{10}+\ldots\right\} .
\end{gathered}
$$

Equation (6) is a large sample approximation due to Kendall (1949) and David, Kendall \& Stuart (1951). As we shall see below, it does not appear to be very accurate when the sample size is as small as 10 . Turning to $r_{K}$, we have

$$
\begin{gathered}
\mathscr{E}\left(r_{K}\right)=\frac{2}{\pi} \sin ^{-1} \rho \quad \text { (Greiner, 1909), } \\
\operatorname{var}\left(r_{K}\right)=\frac{2}{n(n-1)}\left[1-\left(\frac{2}{\pi} \sin ^{-1} \rho\right)^{2}+2(n-2)\left\{\frac{1}{9}-\left(\frac{2}{\pi} \sin ^{-1} \frac{1}{2} \rho\right)^{2}\right\}\right] \quad \text { (Esscher, 1924). }
\end{gathered}
$$

As far as we are aware, no results are available for the higher moments or cumulants of $r_{S}$ or $r_{K}$, but Sundrum (1953) showed how the third and fourth moments of $r_{K}$ might be obtained in the general case. He also used some random sampling results to give empirical values for these moments, assuming underlying normal correlation, in the single case $\rho=1 / \sqrt{ } 2$.

As can be seen from equations (6) and (8), the standard deviations of $r_{S}$ and $r_{K}$ change with $\rho$. Further, as might be anticipated from the parallel case of the product moment correlation coefficient $r_{x y}$, the shape of the sampling distributions are found to change with $\rho$. Thus, when we get away from the problem of using rank correlation coefficients in tests of independence, we at once run into difficulties. The lack of results for dependent rankings has made it difficult to compare the relative merits of different rank coefficients in detecting dependence, nor has it been possible to use these coefficients for a comparison of correlation in different populations. If we accept the underlying bivariate normal struc- 
ture, then we are faced with the distributional problem; if we do not accept this, then we have also to look for a simple definition of non-parametric dependence.

\section{(1·3) The present results and their bearing on these difficulties}

While we do not claim to have solved all these difficulties we hope, in this paper, to have compiled evidence which shows that the problem is capable of a simple solution provided the rankings arise from the class of population models specified below. We proceed as follows:

(A) We start with rankings generated by sampling from a bivariate normal parent with correlation $\rho$. With the help of extensive sampling experiments backed by analytical approximation, we show that if $n$ is not too large the $z$-transforms

$$
z_{S}=\tanh ^{-1} r_{S}=\frac{1}{2} \log _{e} \frac{1+r_{S}}{1-r_{S}}, \quad z_{K}=\tanh ^{-1} r_{K}
$$

are approximately normally distributed with variances nearly independent of $\rho$. In fact

$$
\operatorname{var}\left(z_{S}\right) \sim \frac{1 \cdot 060}{n-3}, \quad \operatorname{var}\left(z_{K}\right) \sim \frac{0.437}{n-4} .
$$

The expectation of $z_{K}$ can be expressed approximately as a simple function of $\rho$, making use of the expressions for $\mathscr{E}\left(r_{K}\right)$ and $\operatorname{var}\left(r_{K}\right)$ given in (7) and (8). The approximation to the expectation of $z_{S}$ is less satisfactory in small samples owing to the inadequacy of the expression (6) for $\operatorname{var}\left(r_{s}\right)^{*}$. It should be noted, however, that, just as in using the $z$-transformation for $r_{x y}$, a knowledge of the precise expectation of the transformed variable is not necessary in a number of the test procedures that become available.

(B) The results in A can clearly be extended to a much wider class of parental distributions. If we start from a bivariate normal distribution of $x, y$ and introduce new variates $X=f(x), Y=g(y)$, the rankings of $X$ and $Y$ will clearly be identical with those of $x$ and $y$ provided the functions $f$ and $g$ are monotonic. Thus, the simple results under A will also apply to rankings generated by the wider class of bivariate distributions of $X, Y$. Conversely, starting from any bivariate distribution $\phi(X, Y)$ we can always find monotonic transformations $X=f(x), \dot{Y}=g(y)$ to standardized normal variates $x$ and $y$. The resulting bivariate distribution $\psi(x, y)$ will not necessarily be bivariate normal, but we think it likely that in practical situations it would not differ greatly from this form. $\dagger$ This is a field in which further investigation would be of considerable interest.

(C) Summarizing the results of A and B, we may state that if the rankings are generated by one of a wide class of distributions of paired variables $\ddagger X, Y$, then the $z$ transforms of the rank correlation measures $z_{S}, z_{K}$ can be regarded as normal variates with variances dependent only on the sample size, and given approximately in equations (10). Further, within this class of bivariate populations, either of the $z$ transforms is an unbiased estimate of a function of the correlation $\rho$. This is the correlation in the bivariate distribution obtained after distortion to normality of the marginal distribution of $X$ and $Y . \rho$ may be regarded as a non-parametric measure of dependence. Without the need to specify $\rho$, simple tests of

* The approximation to the expectation of $z$ contains the variance of $r$; see equation (13) below.

$\dagger$ Johnson (1949) considered a particular case of surfaces having the property of being convertible into the bivariate normal form through the application of his $S_{B}$ and $S_{U}$ transformations to the marginal distributions.

$\ddagger$ It is of course realized that other models of non-parametric dependence have been suggested in which the ranks are not generated by a parental bivariate distribution. Such models are not considered here. 
significance may be applied to the $z$ values to determine whether two or more samples are likely to have come from populations with a common $\rho$.

(D) Within these conditions it is possible to make approximate comparisons of the relative merits of the rank coefficients $r_{S}$ and $r_{K}$ (and later we hope of $r_{F}$ ). In particular, we may compare their power in detecting differences in population $\rho$ values.

\section{The experimental distributions of Spearman's and Kendall's coefficients}

$(2 \cdot 1)$ The distributions of $r_{S}$ and $r_{K}$

The experimental sampling made full use of the 25,000 sets of correlated normal deviates referred to in $\S 1 \cdot 1$. Thus, we had 2500 samples with $n=10,833$ with $n=30$ and 500 with $n=50$. For each value of $n$ we had samples from nine bivariate normal populations, namely those with $\rho=0 \cdot 1(0 \cdot 1) 0 \cdot 9$. The samples of 10,30 and 50 were independent in the sense that the 25,000 cards containing the basic data were re-shuffled between each of the three experiments. The basic calculations for our study were all carried out in the Mathematics Division of the National Physical Laboratory. The samples were formed and ranked on the Division's punched card installation under the supervision of Miss M. U. Thomas. She was responsible, also, for the calculation of all the values of $S_{S}$ (of equation (1)) and $S_{F}$ (the numerator on the right-hand side of equation (4)) and for that of $P_{K}$ (of equation (3)) for samples of size 10. The values of $P_{K}$ for samples of sizes 30 and 50 were obtained on the Deuce digital computer by Mr T. Vickers and Mr B. W. Munday. An account of the methods used will be given in a later paper; we plan also to print the observed frequency distributions corresponding to the various coefficients.

Comparison of the observed mean values of $r_{S}$ with the theoretical values of equation (5) and of the means and variances of $r_{K}$ with equations (7) and (8) is only useful as a check on the representative character of the random samples. This check has been made and passed satisfactorily; the observed values are not reproduced here. Examination of the variance of $r_{S}$ is however necessary, equation (6) giving an approximation only to the true value.

$(2 \cdot 2)$ The variance of $r_{S}$

The Kendall formula (6) does not give the correct values of $1 /(n-1)$ and 0 to $\operatorname{var}\left(r_{S}\right)$ when $\rho=0$ and 1, respectively. A purely empirical adjustment is obtained by substituting $n-1$ for $n$ as divisor and adding a term $+0.019785 \rho^{12}$ which reduces the variance to zero when $\rho=1$, so that we have

$$
\begin{array}{r}
\operatorname{var}\left(r_{S}\right)=\frac{1}{n-1}\left\{1-1.563465 \rho^{2}+0 \cdot 304743 \rho^{4}+0 \cdot 155286 \rho^{6}\right. \\
\left.+0 \cdot 061552 \rho^{8}+0.022099 \rho^{10}+0.019785 \rho^{12}\right\} .
\end{array}
$$

Table 1 contains for each of the three sample sizes, $(a)$ the estimated variance from equation $(11),(b)$ the observed variance from the sampling experiment, $(c)$ smoothed values of $(b)$ obtained by a rough graphical process. These last values are made use of in $\S 3 \cdot 2$ below. It will be seen that for $n=10$, the modified Kendall formula (11) gives values which for $\rho \geqslant 0 \cdot 3$ are consistently smaller than the observed values. The theoretical approximation is also too small, but less noticeably so, when $n=30$. It seems clear that for small samples $\operatorname{var}\left(r_{S}\right)$ cannot be accurately expressed as the product of a function of $n$ and a function of $\rho$. Below, when approximating to the variance of $z_{S}=\tanh ^{-1} r_{S}$ we have therefore used the smoothed observed values of $\operatorname{var}\left(r_{S}\right)$ taken from the third columns of Table 1. 
Table 1. Variance of Spearman's $r_{S}$

\begin{tabular}{|c|c|c|c|c|c|c|c|c|c|}
\hline \multirow{2}{*}{$\rho$} & \multicolumn{3}{|c|}{$n=10$} & \multicolumn{3}{|c|}{$n=30$} & \multicolumn{3}{|c|}{$n=50$} \\
\hline & $\begin{array}{l}\text { From } \\
\text { (11) }\end{array}$ & Obs. & $\begin{array}{c}\text { Smoothed } \\
\text { obs. }\end{array}$ & $\begin{array}{c}\text { From } \\
\text { (11) }\end{array}$ & Obs. & $\begin{array}{c}\text { Smoothed } \\
\text { obs. }\end{array}$ & $\begin{array}{c}\text { From } \\
\text { (11) }\end{array}$ & Obs. & $\begin{array}{c}\text { Smoothed } \\
\text { obs. }\end{array}$ \\
\hline 0.1 & $0 \cdot 1094$ & $0 \cdot 1061$ & $0 \cdot 1093$ & 0.0339 & 0.0334 & 0.0342 & 0.0201 & 0.0192 & 0.0203 \\
\hline 0.2 & $0 \cdot 1042$ & $0 \cdot 1041$ & $0 \cdot 1055$ & 0.0323 & 0.0338 & 0.0336 & 0.0191 & 0.0215 & 0.0200 \\
\hline 0.3 & 0.0958 & 0.1002 & $0 \cdot 1002$ & 0.0297 & 0.0321 & 0.0317 & 0.0176 & 0.0192 & 0.0183 \\
\hline 0.4 & 0.0843 & 0.0916 & 0.0923 & 0.0261 & 0.0263 & 0.0273 & 0.0155 & 0.0160 & 0.0165 \\
\hline 0.5 & 0.0701 & 0.0801 & 0.0805 & 0.0218 & 0.0227 & 0.0225 & 0.0129 & 0.0133 & 0.0135 \\
\hline 0.6 & 0.0539 & 0.0638 & 0.0644 & 0.0167 & 0.0181 & 0.0172 & 0.0099 & 0.0110 & 0.0105 \\
\hline 0.7 & 0.0366 & 0.0443 & $0 \cdot 0470$ & 0.0114 & 0.0117 & 0.0117 & 0.0067 & 0.00604 & 0.0065 \\
\hline 0.8 & 0.0199 & 0.0322 & 0.0303 & 0.0062 & 0.00674 & 0.0067 & 0.0037 & $0 \cdot 00348$ & 0.0035 \\
\hline 0.9 & 0.0062 & 0.0125 & 0.0130 & 0.0019 & 0.00241 & 0.0024 & 0.0011 & 0.00111 & 0.0011 \\
\hline
\end{tabular}

\section{The transformation OF THE RANK CORRELATION COEFFICIENTS}

\section{(3・1) The transformation and its justification}

Our object is to find transformations of $r_{S}$ and $r_{K}$ which will give variances approximately independent of $\rho$ and will at the same time make the distributions roughly normal. The basic distributions of $r_{S}$ and $r_{K}$ become increasingly skew as $|\rho| \rightarrow 1$. It is natural that we should consider R. A. Fisher's $z$ transform which proved so successful in the case of the product moment correlation coefficient $r_{x y}$ in normal samples. If we write in general

$$
z=\tanh ^{-1} r=\frac{1}{2} \log _{e} \frac{1+r}{1-r},
$$

then the cumulants of $z$ may be expanded in series in terms of the cumulants of $r$. The leading terms of the expansions for the mean and variance of $z$ are given in equations (13) and (14):

$$
\begin{gathered}
\mathscr{E}(z)=\frac{1}{2} \log _{e} \frac{1+\bar{r}}{1-\bar{r}}+\frac{\bar{r} \kappa_{2}(r)}{\left(1-\bar{r}^{2}\right)^{2}}+\ldots, \\
\operatorname{var}(z)=\frac{\kappa_{2}(r)}{\left(1-\bar{r}^{2}\right)^{2}}+\ldots,
\end{gathered}
$$

where $\bar{r}=\kappa_{1}(r)=\mathscr{E}(r)$.

The distribution of $r$ depends only on the single parameter $\rho$ and it will be seen that to a first approximation the $z$ transformation may be expected to stabilize the variance of a statistic $r$ if the ratio of $\operatorname{var}(r)$ to $\left(1-\bar{r}^{2}\right)^{2}$ is independent of $\rho$, or nearly so. We have given these ratios in Table $2, \bar{r}_{S}, \bar{r}_{K}$ and $\operatorname{var}\left(r_{K}\right)$ being obtained exactly from equations (5), (7) and (8), respectively, and for $\operatorname{var}\left(r_{S}\right)$ we have used the smoothed observed values from Table 1. The ratios are least constant for $n=10$ where, in particular, there is a definite increase for $\rho=0 \cdot 9$. Further useful comment must await the calculation of $\kappa_{3}(r)$ and $\kappa_{4}(r)$ and a fuller study of the expansions for the cumulants of $z$, but in the meantime we have felt no hesitation in going further with the use of the $z$ transforms. 
Table 2. First approximations to the variance of $z_{S}$ and $z_{K}$

\begin{tabular}{|c|c|c|c|c|c|c|}
\hline \multirow{2}{*}{$\rho$} & \multicolumn{3}{|c|}{$\operatorname{var}\left(r_{S}\right) /\left(1-\bar{r}_{S}^{2}\right)^{2 *}$} & \multicolumn{3}{|c|}{$\operatorname{var}\left(r_{K}\right) /\left(1-\bar{r}_{K}^{2}\right)^{2} \dagger$} \\
\hline & $n=10$ & $n=30$ & $n=50$ & $n=10$ & $n=30$ & $n=50$ \\
\hline $0 \cdot 1$ & 0.111 & 0.035 & 0.021 & 0.0618 & 0.0166 & 0.00952 \\
\hline 0.2 & $0 \cdot 112$ & 0.036 & 0.021 & 0.0619 & 0.0166 & 0.00950 \\
\hline 0.3 & 0.115 & 0.037 & 0.022 & 0.0622 & 0.0166 & 0.00947 \\
\hline 0.4 & 0.120 & 0.037 & 0.022 & 0.0627 & 0.0165 & 0.00943 \\
\hline 0.5 & $0 \cdot 124$ & 0.037 & 0.022 & 0.0634 & 0.0165 & 0.00937 \\
\hline 0.6 & $0 \cdot 126$ & 0.037 & 0.023 & 0.0644 & 0.0164 & 0.00930 \\
\hline 0.7 & 0.130 & 0.038 & 0.022 & 0.0662 & 0.0164 & 0.00920 \\
\hline 0.8 & 0.141 & 0.039 & 0.022 & 0.0697 & 0.0164 & 0.00910 \\
\hline 0.9 & $0 \cdot 155$ & 0.043 & 0.022 & 0.0787 & 0.0168 & 0.00905 \\
\hline $\begin{array}{c}\text { Mean } \\
\rho=0 \cdot 1-0.8\end{array}$ & $0 \cdot 1224$ & 0.0370 & 0.0219 & 0.06404 & 0.01649 & 0.00936 \\
\hline
\end{tabular}

* $\operatorname{var}\left(r_{S}\right)$ obtained from smoothing the experimental values.

$\dagger \operatorname{var}\left(r_{K}\right)$ is the correct theoretical value.

Frequency tables of the distributions of $z_{S}$ and $z_{K}$ have been obtained and the following sections are concerned with comments on the mean values and variances obtained from these tables and with the normality of the distributions.

(3.2) The mean values of $z_{S}=\tanh ^{-1} r_{S}$ and $z_{K}=\tanh ^{-1} r_{K}$

In Table 3 we compare

(a) the observed mean values of $z_{S}$ found from the experimental data;

(b) the approximate theoretical value of $\mathscr{E}\left(z_{S}\right)$ given by the first two terms of (13), namely

$$
\mathscr{E}\left(z_{S}\right)=\tanh ^{-1} \bar{r}_{S}+\bar{r}_{S} \operatorname{var}\left(r_{S}\right) /\left(1-\bar{r}_{S}^{2}\right)^{2},
$$

where $\bar{r}_{S}$ is calculated from (5) and $\operatorname{var}\left(r_{S}\right)$ is the smoothed observed value already referred to;

(c) the second or 'corrective term' from the right-hand side of (15).

Owing to the fact that in a few samples of 10 , the rankings of the two variates were in perfect agreement, some values of $r_{S}$ (and $r_{K}$ ) are unity and the corresponding $z_{S}$ (and $z_{K}$ ) become infinite.* The means and variances tabled omit these observations which in any case form a very small part of a distribution of 2500 observations. We first, however, made estimates of the mean and variance of $z$, using the technique for a censored distribution, but the difference in results was not large enough to be of importance. Having regard to the standard errors quoted below the table $\dagger$ it will be seen that the differences between observation and approximate theory are not significant except perhaps in the case of $\rho=0 \cdot 9$. The corrective term is of some importance in small samples with large $\rho$, but is steadily

* This happened in seven cases for $\rho=0.9$, in three cases for $\rho=0.8$ and once for $\rho=0.7$.

$\dagger$ The standard error of $\bar{z}$ is $\sigma_{z} / \sqrt{ } N$, where the averaged values of $\sigma_{z}$ given below Table 5 for $z_{S}$ and Table 6 for $z_{K}$ have been used and $N=2500,833$ and 500, respectively. 
reduced in importance as $n$ increases. In the case of the transformed product moment correlation coefficient a similar, if less important, effect is present. Table 4 gives similar results for the mean values of $z_{K}$, except that in this case the true values of $\operatorname{var}\left(r_{K}\right)$ may be

Table 3. Mean values of $z_{S}$

\begin{tabular}{|c|c|c|c|c|c|c|c|c|c|}
\hline \multirow{2}{*}{$\rho$} & \multicolumn{3}{|c|}{$n=10$} & \multicolumn{3}{|c|}{$n=30$} & \multicolumn{3}{|c|}{$n=50$} \\
\hline & Obs. & $\begin{array}{l}\text { Approx. } \\
\text { theory }\end{array}$ & $\begin{array}{l}\text { Corr. } \\
\text { term }\end{array}$ & Obs. & $\begin{array}{c}\text { Approx. } \\
\text { theory }\end{array}$ & $\begin{array}{l}\text { Corr. } \\
\text { term }\end{array}$ & Obs. & $\begin{array}{c}\text { Approx. } \\
\text { theory }\end{array}$ & $\begin{array}{l}\text { Corr. } \\
\text { term }\end{array}$ \\
\hline 0.1 & 0.094 & 0.097 & 0.010 & 0.097 & 0.096 & 0.003 & 0.096 & 0.096 & 0.002 \\
\hline 0.2 & $0 \cdot 195$ & $0 \cdot 195$ & 0.019 & $0 \cdot 195$ & $0 \cdot 194$ & 0.007 & $0 \cdot 191$ & $0 \cdot 194$ & 0.004 \\
\hline 0.3 & $0 \cdot 304$ & 0.299 & 0.030 & 0.297 & 0.296 & 0.010 & $0 \cdot 297$ & 0.296 & 0.006 \\
\hline 0.4 & 0.416 & 0.409 & 0.042 & 0.410 & $0 \cdot 405$ & 0.014 & 0.406 & 0.405 & 0.008 \\
\hline 0.5 & 0.526 & 0.529 & 0.055 & 0.517 & 0.525 & 0.017 & 0.522 & 0.525 & 0.010 \\
\hline 0.6 & 0.671 & 0.665 & 0.068 & 0.661 & 0.661 & 0.021 & 0.665 & 0.663 & 0.013 \\
\hline 0.7 & $0.842^{*}$ & 0.825 & 0.082 & 0.833 & 0.826 & 0.025 & 0.838 & 0.828 & 0.014 \\
\hline 0.8 & $1.032^{*}$ & $1 \cdot 038$ & $0 \cdot 103$ & $1 \cdot 051$ & 1.043 & 0.030 & $1 \cdot 056$ & 1.047 & 0.016 \\
\hline 0.9 & $1 \cdot 374^{*}$ & $1 \cdot 361$ & 0.131 & $1 \cdot 406$ & $\begin{array}{l}1 \cdot 389 \\
\end{array}$ & 0.038 & $1 \cdot 417$ & $1 \cdot 399$ & 0.019 \\
\hline
\end{tabular}

Approximate theory $=\tanh ^{-1} \bar{r}_{S}+\bar{r}_{S} \operatorname{var}\left(r_{S}\right) /\left(1-\bar{r}_{S}^{2}\right)^{2}$, using $\bar{r}_{S}$ from equation (5) and the smoothed observed $\operatorname{var}\left(r_{S}\right)$.

Corrective term $=$ second term in expression for approximate theory.

Standard errors of observed means: for $n=10$ about $0 \cdot 008$; for $n=30,50$, about 0.007 .

Table 4. Mean values of $z_{K}$

\begin{tabular}{|c|c|c|c|c|c|c|c|c|c|}
\hline \multirow{2}{*}{$\rho$} & \multicolumn{3}{|c|}{$n=10$} & \multicolumn{3}{|c|}{$n=30$} & \multicolumn{3}{|c|}{$n=50$} \\
\hline & Obs. & $\begin{array}{c}\text { Approx. } \\
\text { theory }\end{array}$ & $\begin{array}{l}\text { Corr. } \\
\text { term }\end{array}$ & Obs. & $\begin{array}{c}\text { Approx. } \\
\text { theory }\end{array}$ & $\begin{array}{l}\text { Corr. } \\
\text { term }\end{array}$ & Obs. & $\begin{array}{c}\text { Approx. } \\
\text { theory }\end{array}$ & $\begin{array}{l}\text { Corr. } \\
\text { term }\end{array}$ \\
\hline $0 \cdot 1$ & 0.065 & 0.068 & 0.004 & $0 \cdot 066$ & 0.065 & 0.001 & 0.065 & 0.064 & 0.001 \\
\hline 0.2 & $0 \cdot 135$ & $0 \cdot 137$ & 0.008 & $0 \cdot 131$ & $0 \cdot 131$ & 0.002 & $0 \cdot 128$ & $0 \cdot 130$ & 0.001 \\
\hline 0.3 & $0 \cdot 209$ & 0.209 & 0.012 & $0 \cdot 199$ & $0 \cdot 200$ & 0.003 & $0: 199$ & $0 \cdot 198$ & 0.002 \\
\hline 0.4 & $0 \cdot 289$ & 0.285 & 0.016 & $0 \cdot 275$ & $0 \cdot 273$ & 0.004 & 0.271 & 0.271 & 0.002 \\
\hline 0.5 & $0 \cdot 361$ & 0.368 & 0.021 & 0.346 & 0.352 & 0.005 & $0 \cdot 346$ & $0 \cdot 350$ & 0.003 \\
\hline $0 \cdot 6$ & $0 \cdot 465$ & 0.462 & 0.026 & 0.439 & 0.442 & 0.007 & 0.439 & 0.439 & 0.004 \\
\hline 0.7 & $0.582^{*}$ & 0.574 & 0.033 & 0.551 & 0.549 & 0.008 & 0.550 & 0.545 & 0.005 \\
\hline 0.8 & $0.717^{*}$ & 0.719 & 0.041 & $0 \cdot 692$ & 0.688 & 0.010 & 0.689 & 0.684 & 0.005 \\
\hline 0.9 & $0.961^{*}$ & 0.949 & 0.056 & 0.917 & 0.905 & 0.012 & 0.909 & 0.899 & 0.006 \\
\hline
\end{tabular}

Approximate theory $=\tanh ^{-1} \bar{r}_{K}+\bar{r}_{K} \operatorname{var}\left(r_{K}\right) /\left(1-\bar{r}_{K}^{2}\right)^{2}$, where $\bar{r}_{K}$ and $\operatorname{var}\left(r_{K}\right)$ are derived from. equations (7) and (8).

Corrective term $=$ second term in expression for approximate theory.

Standard errors of observed means: for $n=10$ about $0 \cdot 0055$; for $n=30,50$ about 0.0045 .

* Ignoring the few infinite values. 
E. C. Fieller, H. O. Hartley and E. S. Pearson

Table 5. Observed variance and standard deviation of $z_{S}$

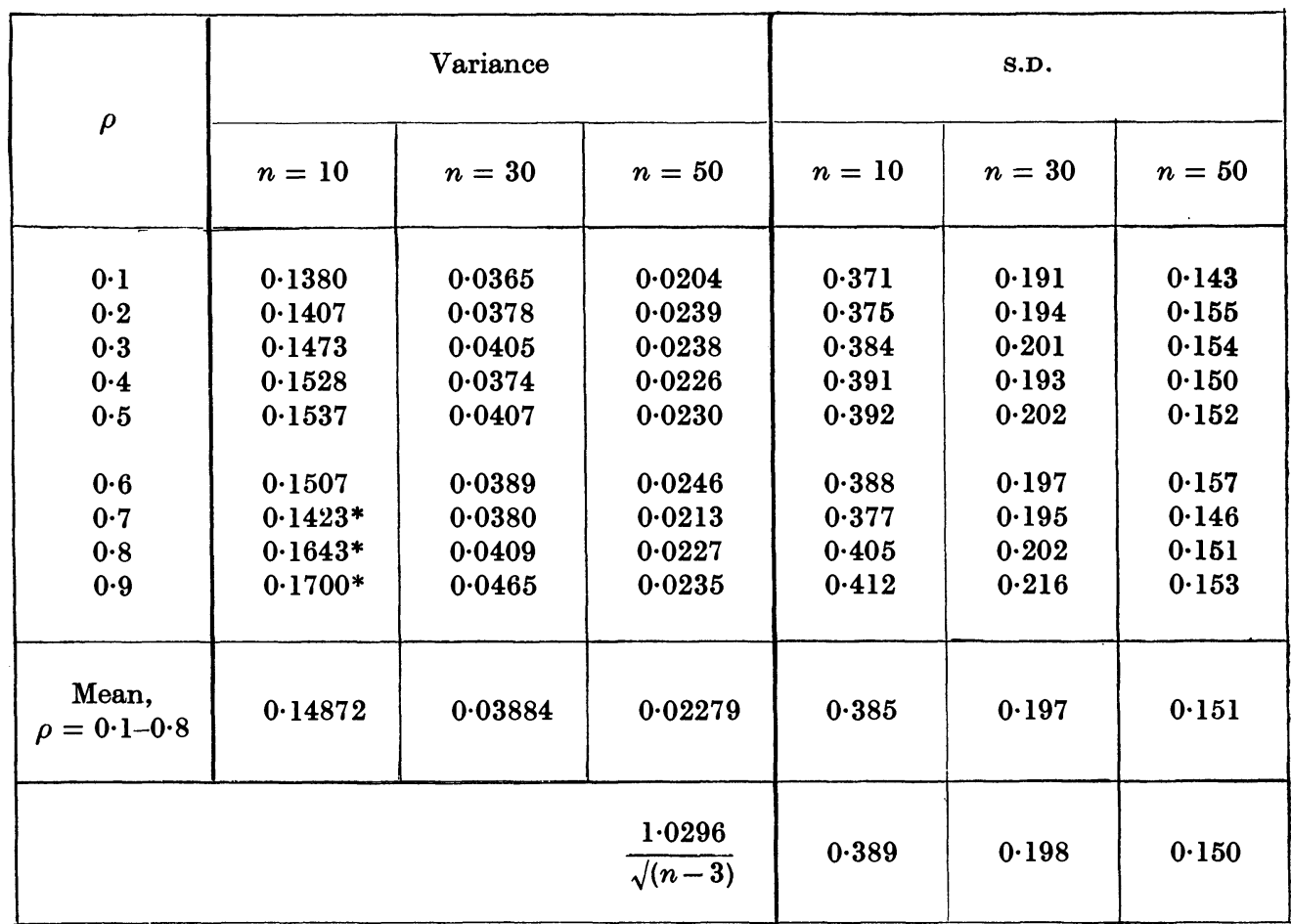

Standard errors of S.D.: $n=10,0.0055 ; n=30,0.0049 ; n=50,0.0047$.

Table 6. Observed variance and standard deviation of $z_{K}$

\begin{tabular}{|c|c|c|c|c|c|c|}
\hline \multirow{2}{*}{$\rho$} & \multicolumn{3}{|c|}{ Variance } & \multicolumn{3}{|c|}{ S.D. } \\
\hline & $n=10$ & $n=30$ & $n=50$ & $n=10$ & $n=30$ & $n=50$ \\
\hline $0 \cdot 1$ & 0.06830 & 0.01700 & 0.00933 & 0.2613 & $0 \cdot 1304$ & 0.0966 \\
\hline $0 \cdot 2$ & 0.06884 & 0.01758 & 0.01074 & $0 \cdot 2624$ & $0 \cdot 1327$ & $0 \cdot 1036$ \\
\hline $0 \cdot 3$ & 0.07290 & 0.01830 & $0 \cdot 01049$ & 0.2700 & $0 \cdot 1353$ & $0 \cdot 1024$ \\
\hline 0.4 & 0.07446 & 0.01639 & 0.00991 & $0 \cdot 2729$ & $0 \cdot 1280$ & 0.0996 \\
\hline 0.5 & 0.07443 & 0.01712 & 0.00966 & $0 \cdot 2728$ & $0 \cdot 1308$ & 0.0983 \\
\hline $0 \cdot 6$ & 0.07384 & 0.01628 & 0.00985 & 0.2717 & $0 \cdot 1276$ & 0.0992 \\
\hline 0.7 & $0.06949 *$ & 0.01514 & 0.00822 & $0 \cdot 2636$ & $0 \cdot 1230$ & 0.0907 \\
\hline $0 \cdot 8$ & $0.08126^{*}$ & 0.01551 & 0.00824 & 0.2851 & $0 \cdot 1245$ & 0.0908 \\
\hline 0.9 & $0.08910^{*}$ & 0.01712 & 0.00780 & $0 \cdot 2985$ & $0 \cdot 1308$ & 0.0883 \\
\hline $\begin{array}{c}\text { Mean, } \\
\rho=0 \cdot 1-0.8\end{array}$ & 0.07294 & 0.01667 & 0.00956 & $0 \cdot 2700$ & $0 \cdot 1290$ & 0.0977 \\
\hline & & & $\frac{0 \cdot 6611}{\sqrt{(} n-4)}$ & $0 \cdot 2699$ & $0 \cdot 1297$ & 0.0975 \\
\hline
\end{tabular}

Standard errors of s.D.: $n=10,0.0038 ; n=30,0.0032 ; n=50,0.0031$.

* Ignoring the few infinite values. 
derived from equation (8). Again, the differences between observation and the approximation appear only to be significant when $\rho=0 \cdot 9$. The corrective term is important for $n=10$ and $\rho$ large; it is smaller in proportion than the corresponding term in the approximation to mean $z_{S}$.

$(3 \cdot 3)$ The variances and standard deviations of $z_{S}$ and $z_{K}$

Tables 5 and 6 contain the observed variances and standard deviations for the transformed variables*. Comparison with Table 2 shows that the first term in the expansions for $\operatorname{var}\left(z_{S}\right)$ and $\operatorname{var}\left(z_{K}\right)$ is definitely not adequate when $n=10$ and still somewhat in defect for the larger samples. These points are brought out by a comparison of the mean values given at the bottom of the tables for the eight cases $\rho=0 \cdot 1$ to $0 \cdot 8$. Apart from the extreme case with $\rho=0 \cdot 9$, the change in the variance of $z$ with $\rho$ is not very great. We shall not attempt now to discuss the changes further. The figures, however, suggest that for most practical purposes if $\rho \leqslant 0.8$ it will be justifiable to assume a constant variance for $z$, for any given sample size not greatly exceeding 50 . The expressions given below are not, however, to be regarded as asymptotic results.

Assuming that we may use the observed mean values given at the bottom of Tables 5 and 6 we may then look for a general empirical expression for the variance of the form

$$
\operatorname{var}(z)=a /(n-b),
$$

where $b$ is an integer. We suggest the use of the following:

For Spearman's coefficient

For Kendall's coefficient

$$
\operatorname{var}\left(z_{S}\right)=\frac{1 \cdot 060}{n-3}, \quad \sigma_{z}=\frac{1 \cdot 03}{\sqrt{(n-3)}} .
$$

$$
\operatorname{var}\left(z_{K}\right)=\frac{0 \cdot 437}{n-4}, \quad \sigma_{z}=\frac{0 \cdot 66}{\sqrt{(n-4)}}
$$

The resulting approximations for the standard deviations of $z_{S}$ and $z_{K}$ when $n=10,30$ and 50 are given at the bottom of the right-hand side of Tables 5 and 6 where they may be compared with the individual sampled values and the means of the latter for $\rho=0 \cdot 1$ to $0 \cdot 8$. We think that except for $\rho>0 \cdot 8$, the approximation can be safely used in tests of significance for $10 \leqslant n \leqslant 50$, provided, of course, that the underlying conditions discussed in $\S 1 \cdot 3$ are applicable to the data.

\section{Normality of the $z$ Distributions; PReliminaRy COMments}

In the case of $n=10$ three difficulties arise in examining the fit of a normal curve to the experimental distributions. In the first place, as mentioned above, in a few samples there was complete agreement between the two rankings so that $r_{S}$ and $r_{K}$ were unity and $z_{S}$ and $z_{K}$ were consequently infinite. These observations have to be omitted in calculating the moments of $z$; alternatively, moments could be estimated using the technique for dealing with truncated observations. Secondly, while possible values for $r_{S}$ and $r_{K}$ are equally spaced, the possible values of $z_{S}$ and $z_{K}$ occur at intervals which increase with the $z$ value. For $n=10$, where the number of permissible values is relatively small, it is a little difficult to know what criterion of normality to adopt. Finally, the distributions of $r_{S}$ exhibit, particularly for low values of $\rho$, the 'saw-edged' character noted by Kendall $(1948$, p. 47$)$ in the case of independence. These factors all make it difficult to know how to

* For $n=30,50$ the variances tabled are $m_{2}$, but for $n=10$ they are $k_{2}=m_{2} N /(N-1)$. 
assess the importance of excessive values of $\chi^{2}$, when comparing the observed distributions with fitted normal curves. Although we have not yet available all the values of $\beta_{2}(z)$, it appears, as in the case of the $z$ transform of the product moment correlation coefficient, that the $z$ distributions are somewhat lepiokurtic $\left(\beta_{2}>3\right)$.

For $n=30$ and 50 we have fitted a certain number of normal curves to the $z$ distributions. The result of applying the $\chi^{2}$ test for goodness-of-fit is summarized in Table 7. Apart from three values of $\chi^{2}$ which are over 30 , the fits appear very reasonable. It is clear that the matter needs further investigation, but we doubt whether even in samples as small as 10, the assumption of a normal $z$ distribution will lead to any serious misinterpretation of a significance test.

Table 7. Normal curve fits to observed distributions of $z_{S}$ and $z_{K}$

\begin{tabular}{|c|c|c|c|c|c|c|c|c|}
\hline \multirow{2}{*}{$\rho$} & \multicolumn{2}{|c|}{$z_{S} ; n=30$} & \multicolumn{2}{|c|}{$z_{S} ; n=50$} & \multicolumn{2}{|c|}{$z_{K} ; n=30$} & \multicolumn{2}{|c|}{$z_{K} ; n=50$} \\
\hline & $x^{2}$ & D.F. & $\chi^{2}$ & D.F. & $x^{2}$ & D.F. & $\chi^{2}$ & D.F. \\
\hline $0 \cdot 1$ & $21 \cdot 1$ & 21 & $16 \cdot 3$ & 19 & - & - & - & - \\
\hline 0.2 & 32.9 & 22 & $20 \cdot 6$ & 20 & - & - & - & - \\
\hline $0 \cdot 3$ & $37 \cdot 8$ & 22 & $20 \cdot 1$ & 20 & - & - & - & - \\
\hline 0.4 & $23 \cdot 7$ & 21 & $23 \cdot 1$ & 20 & - & - & - & - \\
\hline 0.5 & $28 \cdot 7$ & 22 & $13 \cdot 1$ & 20 & $22 \cdot 1$ & 21 & 12.9 & 21 \\
\hline 0.6 & $11 \cdot 1$ & 21 & $17 \cdot 1$ & 20 & - & - & - & - \\
\hline 0.7 & 16.6 & 21 & $29 \cdot 7$ & 19 & - & - & - & - \\
\hline 0.8 & $21 \cdot 9$ & 22 & $15 \cdot 6$ & 19 & $26 \cdot 0$ & 20 & $14 \cdot 7$ & 21 \\
\hline 0.9 & $23 \cdot 0$ & 23 & $15 \cdot 9$ & 20 & $34 \cdot 8$ & 21 & $22 \cdot 3$ & 19 \\
\hline Total & 216.8 & 195 & $171 \cdot 5$ & 177 & 82.9 & 62 & $49 \cdot 9$ & 61 \\
\hline
\end{tabular}

5. The sensitivity of the correlation measures to changes in $\rho$

In broad terms the power of discrimination of any one of the possible correlation measures depends upon the rapidity with which its sampling distributions draw clear of one another as the population $\rho$ changes. If, for example, for a given value of $n$, the distribution of $r_{S}$ for $\rho=0 \cdot 2$ does not sensibly overlap the distribution for $\rho=0 \cdot 8$, then if a single sample of $n$ is drawn from each population a test of significance will always establish a difference in population $\rho$ values. The amount of overlap can of course be seen most directly in the distributions of $r_{S}$ and $r_{K}$ (or $S_{S}$ and $P_{K}$ ) which we hope to publish later.

If the distributions of the $z$ 's were normal with a standard deviation $\sigma_{z}$ which is fixed for a given sample size, the efficiency of discrimination would depend on the way in which the scale of mean $z$ expressed in standard measure (i.e. $\mathscr{E}(z) / \sigma_{z}$ ) opened out as $\rho$ is increased from 0 to 1 . Without assuming a constant $\sigma_{z}$, we can obtain a rough measure of local sensitivity by calculating the ratios $\left(\bar{z}_{2}-\bar{z}_{1}\right) / \sqrt{ }\left(s_{z_{1}}^{2}+s_{z_{2}}^{2}\right)$ of

(a) the differences between pairs of consecutive observed means given in Table 3 (or 4), to

(b) the square roots of the sum of the corresponding pair of observed variances from Table 5 (or 6 ). 
These ratios are given in Table 8 for both Spearman's and Kendall's $z$. We have also given corresponding ratios for the product moment correlation coefficient, taking $\mathscr{E}(z)$ and $\sigma_{z}^{2}$ from the full Fisher expansions as corrected by Gayen (1951, p. 236). Having regard to sampling fluctuations, it is clear that we cannot establish any difference in sensitivity between the two rank coefficients for $n=10$. At $n=30$ and 50 and for $\rho>0 \cdot 6$ the ratio for $z_{K}$ is consistently larger than for $z_{S}$, which suggests a possible advantage for Kendall's coefficient. More detailed examination of this point is however needed. It will be noted, as expected, that the product moment coefficient is throughout more sensitive to changes in $\rho$ than either of the rank coefficients. In all cases for a given difference in $\rho$, the power of discrimination increases with $\rho$.

Table 8. Sensitivity ratios $\left(\bar{z}_{1}-\bar{z}_{2}\right) / \sqrt{ }\left(s_{z_{1}}^{2}+s_{z_{2}}^{2}\right)$ for different coefficients

\begin{tabular}{|c|c|c|c|c|c|c|c|c|c|c|}
\hline \multirow{2}{*}{$\rho_{1}$, } & \multirow{2}{*}{$\rho_{2}$} & \multicolumn{3}{|c|}{$n=10$} & \multicolumn{3}{|c|}{$n=30$} & \multicolumn{3}{|c|}{$n=50$} \\
\hline & & $\begin{array}{l}\text { Product } \\
\text { moment }\end{array}$ & Spearman & Kendall & $\begin{array}{l}\text { Product } \\
\text { moment }\end{array}$ & Spearman & Kendall & $\begin{array}{l}\text { Product } \\
\text { moment }\end{array}$ & Spearman & Kendall \\
\hline $0 \cdot 1$, & 0.2 & 0.205 & $0 \cdot 191$ & $0 \cdot 188$ & 0.383 & $0 \cdot 359$ & 0.352 & 0.501 & 0.452 & 0.447 \\
\hline 0.2 , & $0 \cdot 3$ & 0.214 & 0.202 & $0 \cdot 198$ & 0.399 & $0 \cdot 362$ & 0.359 & 0.523 & 0.489 & 0.484 \\
\hline $0 \cdot 3$, & 0.4 & 0.228 & 0.206 & 0.208 & 0.427 & $0 \cdot 405$ & 0.406 & 0.558 & 0.503 & 0.508 \\
\hline 0.4 , & 0.5 & 0.250 & $0 \cdot 198$ & $0 \cdot 188$ & 0.469 & 0.384 & 0.387 & 0.615 & 0.542 & 0.538 \\
\hline 0.5 , & 0.6 & 0.286 & 0.263 & 0.270 & 0.537 & 0.508 & 0.508 & 0.704 & 0.655 & 0.664 \\
\hline 0.6 , & 0.7 & $0 \cdot 345$ & 0.315 & $0 \cdot 308$ & 0.649 & 0.622 & 0.636 & 0.851 & 0.809 & 0.828 \\
\hline 0.7 , & 0.8 & $0 \cdot 456$ & $0 \cdot 345$ & $0 \cdot 348$ & 0.861 & 0.776 & 0.805 & $1 \cdot 13$ & $1 \cdot 04$ & $1 \cdot 08$ \\
\hline 0.8 & 0.9 & 0.732 & 0.592 & 0.592 & 1.39 & $1 \cdot 20$ & $1 \cdot 24$ & 1.82 & $1 \cdot 68$ & $1 \cdot 74$ \\
\hline
\end{tabular}

\section{Concluding remarks}

Besides putting on record the basic sampling distributions we hope in a further paper to carry our investigations further in a number of directions, in particular to give parallel results for the coefficient $r_{F}$ of equation (4).

We should like to express our great indebtedness to Miss M. U. Thomas and Mr T. Vickers whose work has already been mentioned, to Mrs Esmé Hill, formerly of the Statistical Advisory Unit, Ministry of Supply, and to Mrs Maxine Merrington and Miss Janet Hall of University College London. Finally, we should like to say how much we owe to the cooperation of the Mathematics Division of the National Physical Laboratory for the facilities which made this investigation possible.

\section{REFERENCES}

DANIELS, H. E. (1950). J. R. Statist. Soc. B, 12, 171-81.

David, S. T., Kendall, M. G. \& Stuart, A. (1951). Biometrika, 38, 131-40.

Esscher, F. (1924). Skand. AktuarT'idskr. 7, 201-19.

Freller, E. C., Lewis, T. \& Pearson, E. S. (1955). Correlated Random Normal Deviates. Tracts for Computers, no. XXVI. Cambridge University Press.

Frsmer, R. A. \& YAtes, F. (1938). Statistical Tables for Biological, Agricultural and Medical Research (5th edition, 1957). Edinburgh: Oliver and Boyd. 
GAYEN, A. J. (1951). Biometrika, 38, 219-47.

GrenNer, R. (1909). Z. Math. Phys. 57, 121, 225, 337.

Hoefforng, W. (1951). Proceedings of the Second Berkeley Symposium on Mathematical Statistics and Probability, 83-92. University of California Press.

Jeffreys, Harold (1948). Theory of Probability. Oxford University Press.

Johnson, N. L. (1949). Biometrika, 36, 297-304.

Kandall, M. G. (1948). Rank Correlation Methods (2nd edition, 1955). London: Chas. Griffin and Co., Ltd.

Kendall, M. G. (1949). Biometrika, 36, 177-93.

Mallows, C. L. (1957). Biometrika, 44, 114-30.

Moran, P. A. P. (1948). Biometrika, 35, 203-6.

Moran, P. A. P. (1950). J. R. Statist. Soc. B, 12, 153-62.

SUNDRUM, R. M. (1953). Biometrika, 49, 409-20. 